\title{
A novel natural coating for food preservation: Effectiveness on microbial growth and physicochemical parameters
}

\author{
Marcio Carocho $^{\mathrm{a}, \mathrm{b}}$, Sandrina Heleno ${ }^{\mathrm{a}}$, Paula Rodrigues ${ }^{\mathrm{a}}$, M. Filomena Barreiro ${ }^{\mathrm{a}, \mathrm{b}}$, Lillian Barros ${ }^{\mathrm{a}}$, \\ Isabel C.F.R. Ferreira ${ }^{\mathrm{a}, *}$ \\ ${ }^{a}$ Centro de Investigação de Montanha, (CIMO), Instituto Politécnico de Bragança, Campus de Santa Apolónia, 5300-253, Bragança, Portugal \\ ${ }^{\mathrm{b}}$ Laboratory of Separation and Reaction Engineering - Laboratory of Catalysis and Materials (LSRE-LCM), Bragança Polytechnic Institute, 5301-857, Bragança, Portugal
}

\section{A R T I C L E I N F O}

\section{Keywords:}

Biopolymers

Ham preservation

Microbial growth

Physicochemical parameters

\begin{abstract}
A B S T R A C T
This work describes the development of a sustainable and natural coating solution based on iota-carrageenan and rosemary extract intended to preserve cooked ham through dipping. Upon comparing the physical and microbial load changes between this solution and the traditional plastic wrap along 15 consecutive days, the results showed that in terms of microbial load the samples treated with the developed solution had a lower microbial count. Furthermore, only slight changes in moisture loss and hardness were observed between samples using the two tested preservation strategies. Colour analysis revealed that dipped samples presented reduced lightness, with a tendency to avoid shifting to a blue tone over the 15-days of storage. This work indicates that natural polymers combined with natural antimicrobials and antioxidants can be employed to reduce the dependence on synthetic polymers and offer feasible solutions to be applied at an industrial level.
\end{abstract}

\section{Introduction}

The food industry uses polymeric materials in a large scale, namely polyamide (PA), polyethylene terephthalate (PET), ethylene vinyl alcohol (EVOH), polyvinylidene chloride (PVdC) polypropylene (PP) and polyethylene (PE). Given their widespread use for food preservation, polymers in direct contact with foodstuffs allow migration of their additives and other components (e.g. residual monomers) into food. Plasticizers seem to be the most relevant cases, and the most studied migrating compounds, especially phthalates, adipates, citrates and epoxidized soy bean oil (ESBO), among others. Phthalates, for example, are known as endocrine disruptors, by mimicking naturally occurring hormones, and thus deregulating endocrine functions. Moreover, they can affect children at a pre-natal level, due to maternal exposure. Widespread human and environmental exposure to phthalates has been described, with ingestion being the main route of administration (Stojanoska, Milosevic, Milic, \& Abenavoli, 2017; Toni et al., 2017). Furthermore, phthalates are also associated with erectile dysfunction in human males, along with breast and other cancers, while also being responsible for various deleterious effects in murine models and cell lines (Absalan et al., 2017; Lopez et al., 2017; Rocha et al., 2017; Zuccarello et al., 2018).

Regulation of phthalates and other plasticizers has been increasing, especially in the EU, with the Commission Regulation 10/2011 removing many plasticizers as food contact materials (FCM) or limiting their migration limits. Overall, and even after legislation has reduced the load of plasticizers, concerns still linger, due to a lack of global legislation and imported goods from countries where legislation is less restrictive (Muncke et al., 2017; Nerin, Canellas, \& Vera, 2018).

In recent years, research is being focused on alternative bio-based non-toxic plastics and plasticisers to substitute synthetic counterparts. These alternatives are in line with consumer standards regarding healthier foods and food preservation techniques, while also being sustainable for the environment by reducing the dependence on plastics (Brostow, Lu, \& Osmanson, 2018). Other alternatives are food coatings, that can be made of lighter, cheaper and sustainable polymers, namely polysaccharides such as cellulose, starch, pectin derivatives, alginates, seaweed extracts like carrageenan and agar, but also protein- and lipidbased materials. One great advantage is the ability to mix them with antimicrobials and antioxidants to improve organoleptic properties, reduce microbial load, and increase visual and physical characteristics (Aloui \& Khwaldia, 2016; Dehghani, Hosseini, \& Regenstein, 2018). In recent years, a strong focus has been directed to develop these sustainable coatings, due to their ability to incorporate natural preservatives and antimicrobials from plant extracts, thus reducing the need for food processing steps where these would be added to the food

\footnotetext{
* Corresponding author.

E-mail address: iferreira@ipb.pt (I.C.F.R. Ferreira).
} 
itself (Ganiari, Choulitoudi, \& Oreopoulou, 2017; Portillo et al., 2018). Plant extracts from Thyme have been used on chitosan edible films to reduce microbial load and excerpt their antioxidant properties on cooked cured ham, while coatings of whey protein were incorporated with Bifidobacterium and Lactobacillus to achieve the same goal, namely to extend shelf-life and reduce spoilage bacteria. In addition, various antimicrobial carbohydrate coatings have been tested against Listeria monocytogenes, while pomegranate peel extracts have been used to be incorporated into chitosan, to fight off Penicillium digitatum in post harvested oranges (Jiang, Neetoo, Chen, \& Haiqiang, 2011; Kharchoufi et al., 2018; Pereira, Soares, Monteiro, Gomes, \& Pintado, 2018; RuizNavajas et al., 2015).

In this study, the microbial load and physical characteristics (moisture, texture and colour) of fresh ham, coated with iota carrageenan as a bio-based edible coating added with natural aqueous extract of Rosmarinus officinalis (Rosemary), are compared to a food-grade plastic wrap, being the results reported throughout a storage time of 15 days at $5{ }^{\circ} \mathrm{C}$.

\section{Materials and methods}

\subsection{Reagents}

Iota-carrageenan was acquired from Alfa Aesar (Havernhill MA, USA), calcium chloride and ascorbic acid were acquired from PanReac (Barcelona, Spain), $\alpha$-tocopherol and glycerol from Thermo Fischer Scientific (Hampton, NH, USA). The rosemary plants were bought from a Portuguese dried plants retailer, "Cantinho das Aromáticas". Water was treated with a Milli-Q water purification system (TGI Pure Water Systems, Greenville, SC, USA). Peptone water (PW), Plate Count Agar (PCA), Violet Red Bile Lactose Agar (VRBLA), 4-methylumbelliferylbeta- $D$-glucuronide (MUG), Dichloran Rose Bengal Chloramphenicol Agar (DRBC) supplemented with $0.1 \%$ chloramphenicol, Man, Rogosa and Sharpe agar (MRS), and Baird Parker Agar (BP) were from Liofilchem, Italy. All remaining, or non-referred chemicals were of analytical grade and acquired from scientific retailers.

\subsection{Samples}

The Rosemary samples were obtained from the enterprise "Cantinho das Aromáticas", organic farmers from Vila Nova de Gaia, Portugal. The samples consisted of the dried leaves of rosemary which after reception at the laboratory were reduced to a 20 -mesh powder.

\subsection{Preparation of the bio-based coating solution}

For this, $25 \mathrm{~g} / \mathrm{L}$ of the ground aerial parts were added to boiling distilled water $\left(\sim 100^{\circ} \mathrm{C}\right)$, mixed and left to boil for $5 \mathrm{~min}$. After this, the heating was turned off and the infusion left an extra $5 \mathrm{~min}$ before being filtered through a Whatman №4 paper filter. Finally, the filtrate was frozen, lyophilized and ground to a $\sim 20$ mesh powder. The obtained yield of the extraction was $20 \%$. A previous study by the authors attests the quality of the rosemary used in this study, namely the presence of the bioactive compounds, such as rosmarinic acid (major compound found), yunnaneic acids, luteolin glycoside derivative, and a caffeic acid derivative, among others (Ribeiro et al., 2016).

Regarding the coating solution, it consisted of a dipping system, where the ham portions were dipped in for approximately $15 \mathrm{~s}$. Initially, the solution consisted of deionized water with diluted rosemary extract $(0.5 \%, \mathrm{w} / \mathrm{v})$ kept under vigorous stirring, to which ascorbic acid (antioxidant) and calcium chloride (filler) were added at 0.1 and $1 \%(\mathrm{w} / \mathrm{v})$, respectively. At the same time $\alpha$-tocopherol was diluted in $10 \mathrm{~mL}$ of ethanol, protected from light, and then added to the main solution. Iota-carrageenan ( $1 \%$ ) was then added to the mixture, followed by $3 \%$ of glycerol as soon as the carrageenan had become completely dissolved in the medium. The use of alpha-tocopherol is related to its great effect as an antioxidant in lipophilic mediums, while ascorbic acid is added to regenerate the alpha-tocopherol after it has been oxidized by free radicals formed on the food surface, while also being a potent hydrophilic antioxidant (Carocho \& Ferreira, 2013). Iota carrageenan was used for its higher elasticity and clear gels when compared to the other two carrageenan types, namely lambda and kappa. Iota carrageenan also jellifies in the presence of cations, hence the use of calcium chloride as a filler. Furthermore, calcium chloride is proven to have synergistic effects with biopolymers like chitosan, on the enhancement of textural properties of fruits and other foods, making it the ideal candidate for filling purposes in this coating solution. Glycerol was used to reduce the lumpiness of the solution by improving its continuity and smoothness (Chong, Lai, \& Yang, 2015; Tavassoli-Kafrani, Shekarchizadeh, \& Masoudpou-Behabadi, 2016).

\subsection{Experimental design}

To uncover the effects of different preservation treatments, 4 sets of hams were prepared (in triplicate) from one piece of vacuum-packed cooked ham, obtained from Sonae, MC (a nationwide Portuguese retail company). Each set consisted of identical pieces of ham with approximately $2 \mathrm{~cm}^{3}$ and average weight of $30 \pm 4 \mathrm{~g}$. Set 1 was the "Control", in which no preserving treatment was performed; set 2 consisted of the "Dipping" set, in which the samples were dipped into the solution described in section 2.2. for $15 \mathrm{~s}$; set 3 underwent a "Wrapping" with commercial plastic wrap, and finally set 4 underwent "Both" treatments, a dipping followed by wrapping procedure. Wrapping was performed by placing the ham piece on a plastic wrap square and then folding the square over the ham until it was completely covered. Immediately after treatments, all samples were placed in trays and stored at a constant temperature of $5{ }^{\circ} \mathrm{C}$ in a cold storage room $\left(5^{\circ} \mathrm{C}\right)$. Seven different storage times, measured in days, were used to monitor the changes in physical (texture properties and external colour) and microbial load, namely T0 (immediately after treatments), T3, T5, T7, T10, T12 and T15.

\subsection{Microbiological analysis}

\subsubsection{General sample preparation}

The preparation of samples for microbiological analysis followed the procedure described in the International Organization for Standardization (ISO) 6887-1:2003. Ham samples $(30 \pm 4 \mathrm{~g}$ ) were mixed with $90 \mathrm{~mL}$ of peptone water (PW) in stomacher bags and further homogenized in a stomacher equipment (ECN 710-0873, Italy) for $1 \mathrm{~min}$ at 300 units. The obtained suspensions were further diluted to obtain dilutions from $10^{-1}$ to $10^{-5}$. Each dilution was analysed in duplicate.

\subsubsection{Microorganisms analysis}

Aerobic plate count (APC): $1 \mathrm{~mL}$ of each prepared suspension was mixed with $20 \mathrm{~mL}$ of Plate Count Agar (PCA) by the pour plate method, in duplicate $(\mathrm{LOQ}=1 \log \mathrm{UFC} / \mathrm{g}$ ). The plates were further incubated in reversed position at $30^{\circ} \mathrm{C}$ for $72 \mathrm{~h}$ and counted according to ISO 4833 2:2013.

Coliforms and E. coli: For the coliforms counting, $1 \mathrm{~mL}$ of each suspension was mixed with $20 \mathrm{~mL}$ of Violet Red Bile Lactose Agar (VRBLA), by the plate method, in duplicate (LOQ $=1 \log$ UFC/g). For E. coli determination, the medium was supplemented with 4-methylumbelliferyl-beta-D-glucuronide (MUG). The plates were further incubated in reversed position at $30^{\circ} \mathrm{C}$ for $48 \mathrm{~h}$ and counted according to ISO 4832:2006 standard.

Yeasts and molds: $0.2 \mathrm{~mL}$ of each suspension was spread in petri dishes containing $20 \mathrm{~mL}$ of Agar Dicloran Rosa Bengala Cloranfenicol Base (DRBC), in duplicate ( $\mathrm{OOQ}=1.7 \mathrm{log} \mathrm{UFC} / \mathrm{g}$ ). The plates were further incubated in upright position at $25^{\circ} \mathrm{C}$ for: $72 \mathrm{~h}$ for yeast counting and $120 \mathrm{~h}$ for mould counting, according to ISO 21527-2:2008 standard. 


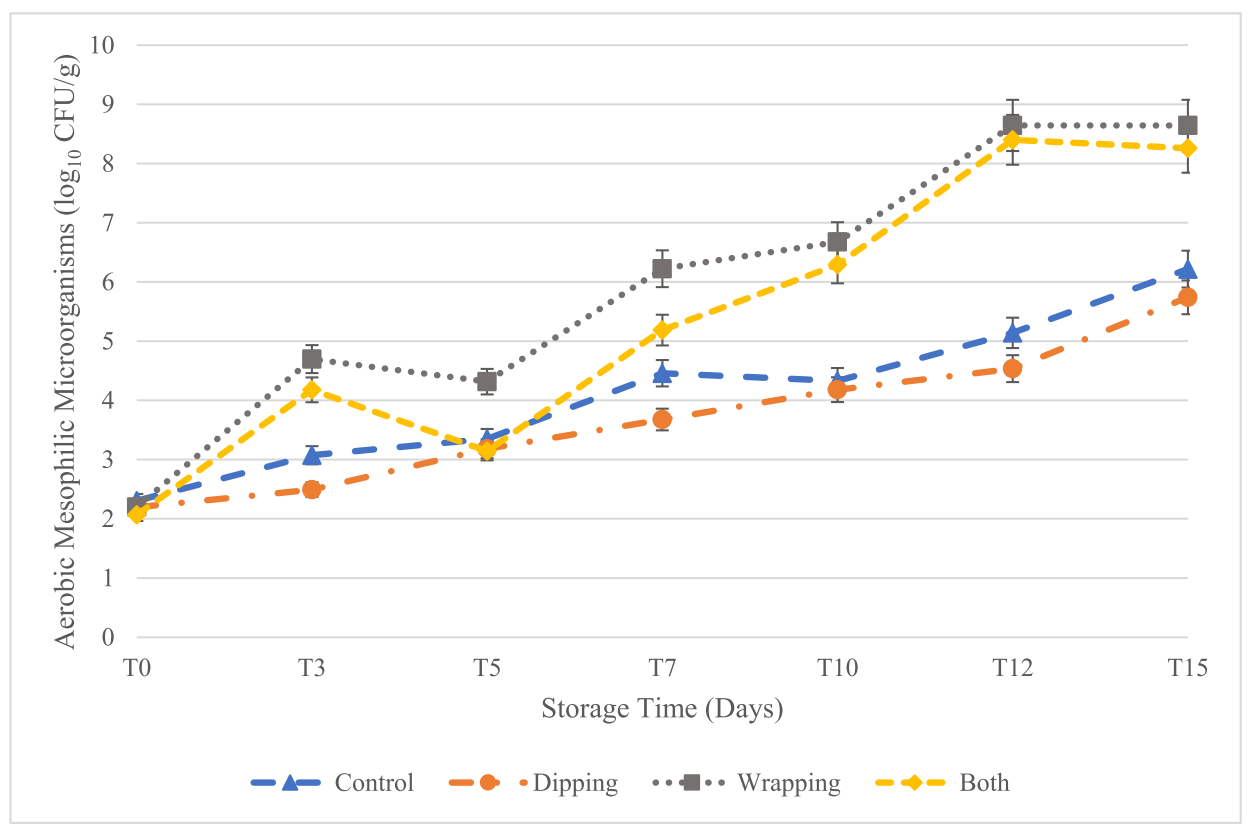

Fig. 1. Effect of the different treatments in the development of the aerobic mesophilic microorganisms over 15 days of storage, plotted in a 10-base logarithmic scale.

Psychrotrophic bacteria: $1 \mathrm{~mL}$ of each suspension was mixed with $20 \mathrm{~mL}$ of Man, Rogosa and Sharpe agar (MRS), by the plate method, in duplicate. The medium was allowed to solidify, and another $5 \mathrm{~mL}$ of medium was added to create anaerobiosis (LOQ $=1 \log \mathrm{UFC} / \mathrm{g}$ ). The plates were further incubated in upright position at $22^{\circ} \mathrm{C}$ for 5 days and counted according to the International Organisation for Standardization (ISO) number 4832:2006 (ISO, 1998).

Staphylococcus aureus: $0.2 \mathrm{~mL}$ of each suspension was spread in petri dishes containing $20 \mathrm{~mL}$ of Baird Parker Agar (BP), in duplicate $(\mathrm{LOQ}=1.7 \log \mathrm{UFC} / \mathrm{g})$. The plates were further incubated in reverse position at $37^{\circ} \mathrm{C}$ for $24 \mathrm{~h}$ for yeast counting, and $120 \mathrm{~h}$ for mould counting, according to ISO 6888-1 (ISO, 1999).

\subsection{Texture and colour analysis}

\subsubsection{Texture}

Texture analysis was carried out on a Stable Micro Systems' (Vienna Court, Godalming UK) TA.XT Plus Texture Analyser with a $30 \mathrm{Kg}$ load cell, using the $\mathrm{P} / 4545 \mathrm{~mm}$ aluminium cylinder probe. A Texture Profile Analysis (TPA) was carried out on the samples with $5 \mathrm{~mm} / \mathrm{s}$ as the preand post-test speed, and $3 \mathrm{~mm} / \mathrm{s}$ as the test speed. The target mode was set to "strain" and causing $25 \%$ strain to the samples for 5 consecutive seconds, while the trigger was set to "force" and starting the measurement at $50 \mathrm{~g}$ of force. After the analysis, a macro was performed in order to measure various dimensions of texture, namely hardness, adhesiveness, springiness, cohesiveness, chewiness and resilience. The texture results were achieved through the Exponent program, proprietary of Stable Micro Systems. The moisture variation was calculated based on water loss during the storage time by weighing the samples at the different storage times.

\subsubsection{Colour analysis}

For each sample and storage time, the external colour was measured in six different points of the surface of the ham piece. This was performed with a portable CR400 colorimeter from Konica Minolta (Chiyoda, Tokyo, Japan) with the $\mathrm{D}_{65}$ illuminant, a standard illuminant defined by the International Commission on Illumination (CIE) which represents the midday light in Europe (daylight illuminant). The CIE $\mathrm{L}^{*}$ $a^{*} b^{*}$ colour space of 1976 was used, with L* representing lightness, a* representing redness (red-green), and $b^{*}$ representing yellowness (yellow-blue), with a $10^{\circ}$ observer angle and $8 \mathrm{~mm}$ aperture.

\subsection{Statistical analysis}

All data was expressed as means \pm standard deviation, and assays where carried out in triplicate. For the microbial data, after the confirmation of the homoscedasticity of the samples, a simple analysis of variance (ANOVA) was performed with a Tukey test to classify the differences. Regarding the physical parameters, texture and colour, an ANOVA with type III sums of squares was carried out with the SPSS software (IBM Corporation, Armonk, USA). Using a multivariate general linear model, the dependent variables were analysed through a 2way ANOVA, with the factors "Treatment" (T) and "Storage Time" (ST). When a significant interaction was detected for both the dependent factors ( $\mathrm{T}$ and ST), they were evaluated simultaneously by the estimated marginal means. If, on the contrary, no significant interaction was detected, the means were compared using Tukey's multiple comparison test, relying on the previous assessment of the equality of variances through the Levene's test. All statistical operations were performed at a $5 \%$ significance level.

\section{Results and discussion}

\subsection{Microbial counts}

Microbial counts obtained for the aerobic mesophilic microorganisms (APC), coliforms, lactic acid bacteria (LAB) and yeasts in cooked ham stored at $5{ }^{\circ} \mathrm{C}$ for 15 days are shown in Figs. 1-4 (plotted in a tenbase logarithmic scale of Colony Forming Units per gram, CFU/g). No molds or coagulase-positive Staphylococcus sp. were detected in any of the treatments regardless of time of storage.

Regarding the APC's (Fig. 1), all treatments resulted in increased counts throughout the storage period, with counts for "Control" and "Dipping" significantly lower (p < 0.05) than "Wrapping" and "Both". The water retention between the plastic wrap and the ham surface caused by wrapping seems to be the cause for the higher increase of these microorganisms during storage, when compared with the nonwrapped samples. Moreover, the "Dipping" treatment showed to be slightly more effective $(p>0.05)$ in controlling the development of aerobic microbes than the "Control", which can be attributed to the 


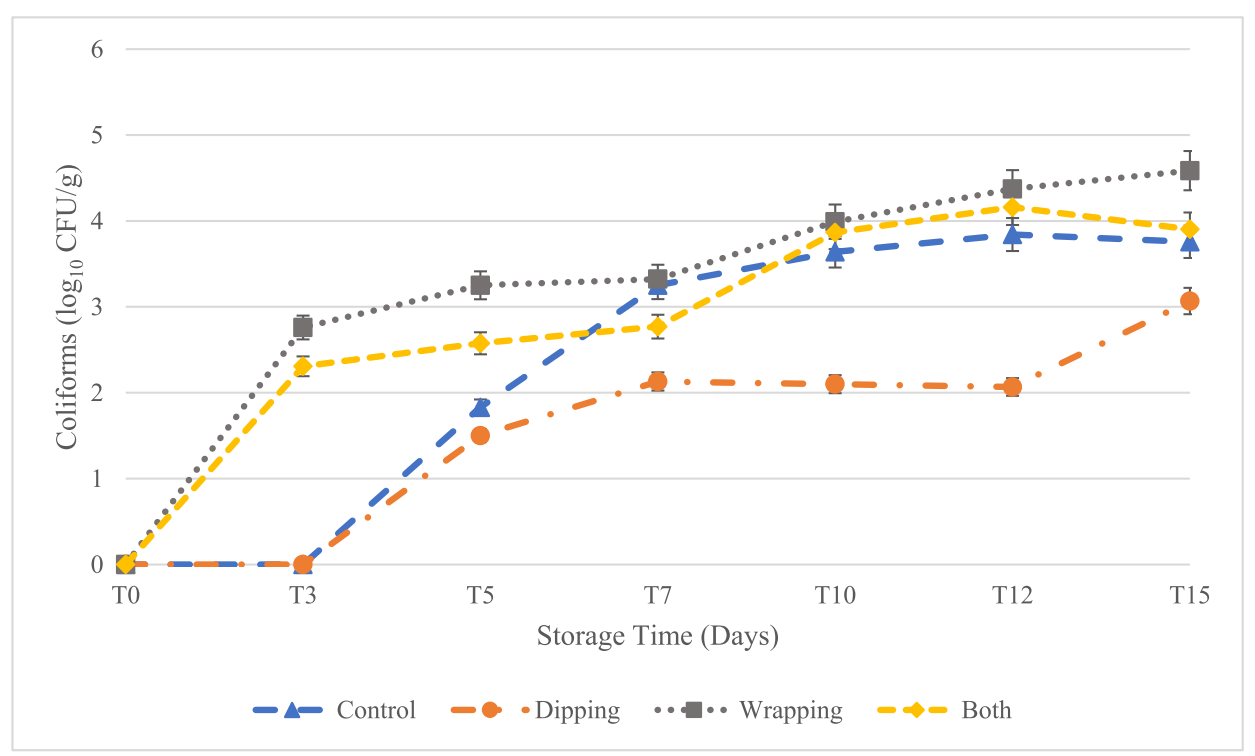

Fig. 2. Effect of the different treatments in the development of coliforms over 15 days of storage, plotted in a 10-base logarithmic scale.

antimicrobial effect of the rosemary extract, and other antioxidant components of the coating solution. These findings are in line with the study of Viuda-Martos, Ruiz-Navajas, Fernández-López, and PéresÁlvarez (2010), that showed that after twelve and twenty-four days of storage, vacuum-packed mortadella coated with rosemary and thyme extracts had lower APC counts than control samples.

Fig. 2 plots the development of coliforms, which are used as process hygiene indicators. For the first three days of storage, both the "Control" and "Dipping" samples showed no counts of these contaminants, while the other treatments had an increase over this time period, with "Wrapping" samples showing the highest counts. At five days, "Control" samples started following the same increasing trend as did "Wrapping" and "Both" samples. From the tenth day onwards, the "Dipping" treatment showed significantly lower counts $(\mathrm{p}<0.05)$ than the other treatments. This assay proves the efficacy of the tested coating as a protector and inhibitor of coliforms development over time, when considering longer (abusive) storage periods (seven to fifteen days).
This is probably due to the antimicrobial properties of rosemary extract, which has recurrently been described in literature, aided by ascorbic acid (Andrade, Ribeiro-Santos, Bonito, Saraiva, \& Sanches-Silva, 2018; Gonçalves et al., 2019). Furthermore, in the European Food Safety Authority's (EFSA) report of rosemary extract it has also been recognized as a food preservative, which corroborates its use as a food coating component (EFSA, 2008). As previously described for APC, the moisture retained between the ham surface and the plastic wrap seems to have had an important influence in the higher amount of these contaminants for the "Wrapping" and "Both" samples, although the antimicrobial capacity of the extract helped keep a significantly lower $(\mathrm{p}<0.05)$ amount in the "Both" samples.

Fig. 3 displays the development of psychrotrophic LAB. These Grampositive fermenting bacteria are able to develop under refrigerated conditions and cause food spoilage. They are useful indicators of commercial quality and of certain products' shelf-life. On the third day of storage, LAB were not detected in "Control" and "Dipping" samples,

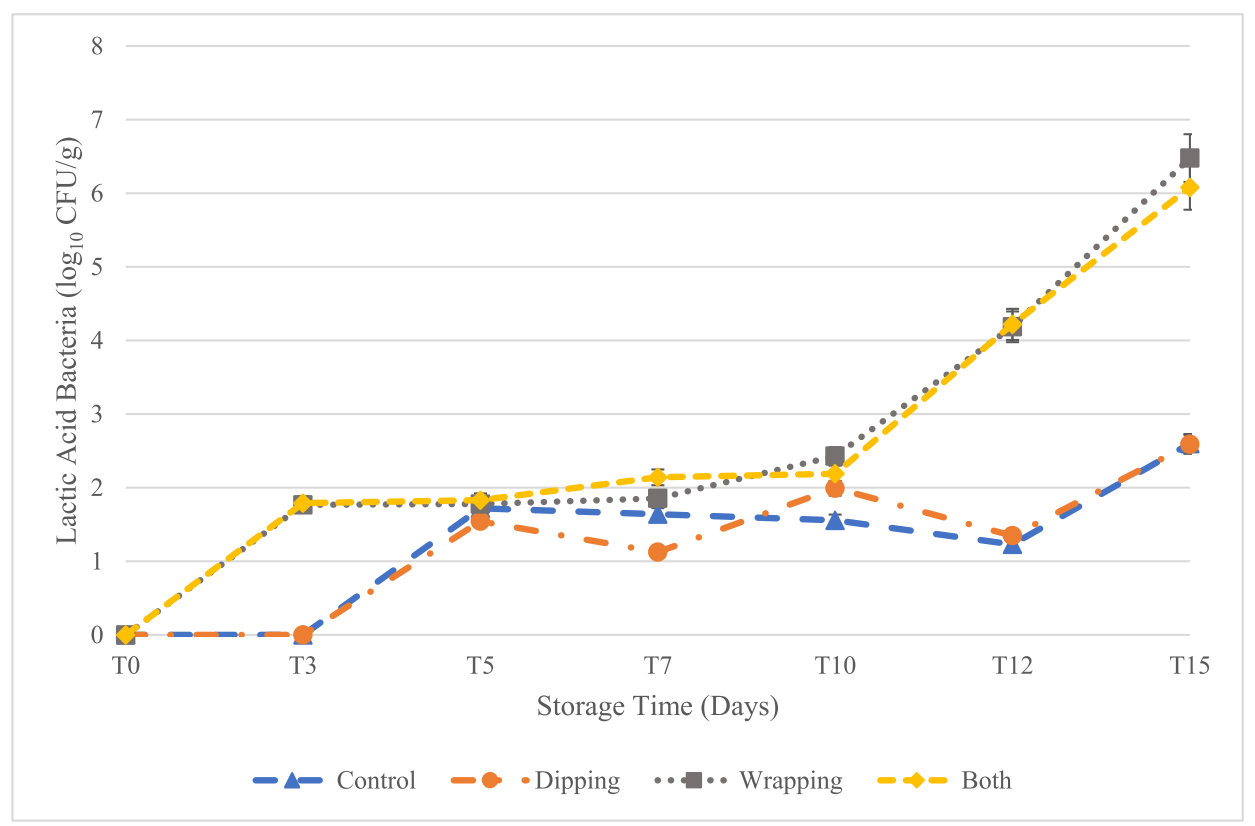

Fig. 3. Effect of the different treatments in the development of the lactic acid bacteria over 15 days of storage, plotted in a 10-base logarithmic scale. 


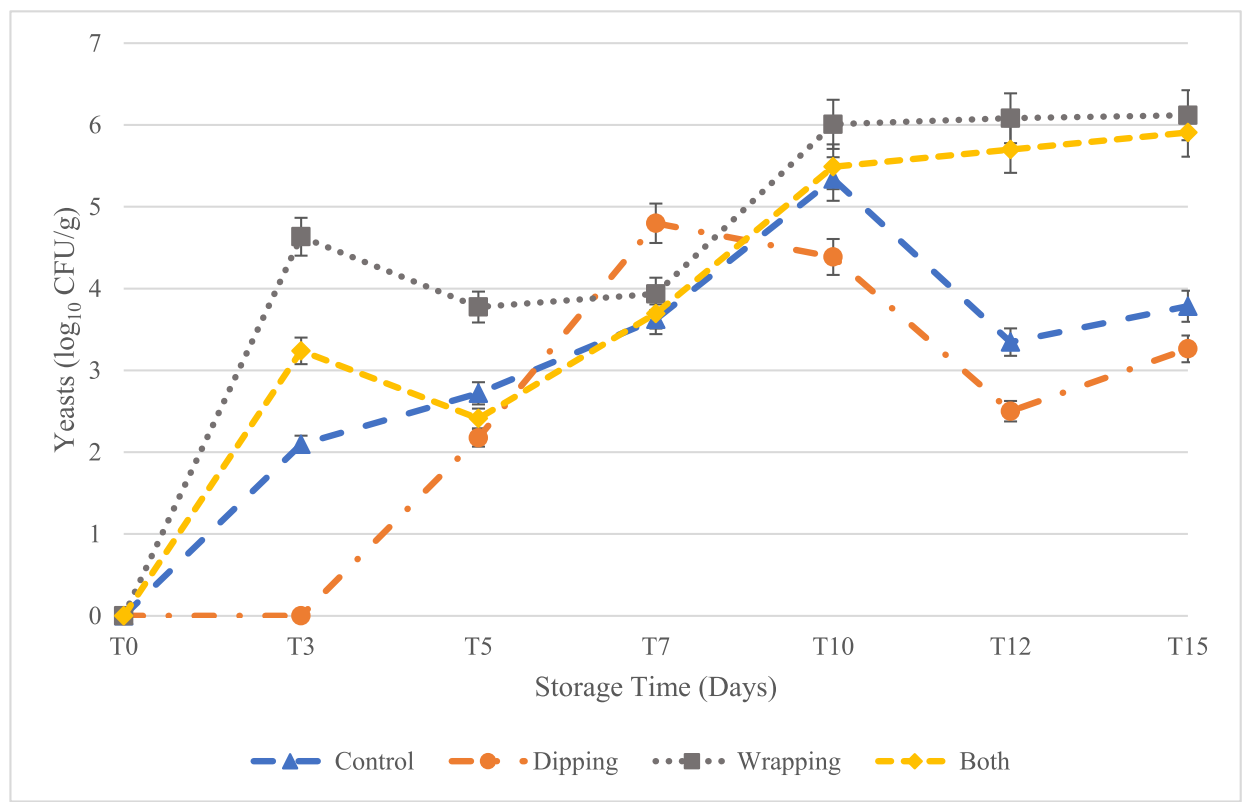

Fig. 4. Effect of the different treatments in the development of yeasts over 15 days of storage, plotted in a 10-base logarithmic scale.

while wrapped samples (with or without coating) showed development of these contaminants, with no significant differences $(\mathrm{p}<0.05)$ between them. From day 5 to day 10, coated samples showed the lowest number of LAB, but not significantly different from the other treatments. Along the storage time, the samples with plastic wrapping always showed higher amounts of these bacteria, with significant difference after the tenth day of storage, as depicted in Fig. 3. From there onwards, in terms of the "Control" and "Dipping" samples, although having slight variations, no statistical differences could be sought between them. In 2010, Viuda-Martos et al. also tested rosemary and thyme extracts on lactic acid bacteria in mortadella and concluded that after twelve and twenty-four days, the extracts were effective in reducing LAB loads, which is in agreement with the findings of this work, although these contaminants were the least affected by the coating solution. The observed differences in the results can be attributed to the different extraction procedures and further alterations in the composition of the obtained rosemary extracts.

Finally, in Fig. 4 the growth of yeasts is plotted over the fifteen days of storage. The dipping treatment resulted in the lowest yeast counts throughout the whole storage period except for day 7, proving itself as the most effective treatment in controlling yeast growth $(\mathrm{p}<0.05$; except day 7).

Accounting for the four studied microbial growths, it seems clear that the amount of moisture retained in the hams overtime had an important role on the development of these contaminants, with a clear enhancement of their growth in samples with higher moisture, namely "Wrapping" and "Both". Still, in some cases, the samples that were exposed to the dipping solution, "Dipping" and "Both", showed a higher capacity of controlling microbial growth than their counterparts, namely "Wrapping" from the fifth day onwards for the APC's, and during the whole storage time for the coliform growth, which also saw a better control of their development from the tenth to the twelfth day for "Dipping" in relation to "Control". For the LAB analysis, there were few statistical differences among the samples, thus being the assay where the dipping solution seemed to have the lowest impact. In terms of yeasts, the best period of activity of the dipping solution seems to be in the first week, although after the tenth day, it was the best treatment to control the growth of these spoilage contaminants. Other studies have pointed out the moderate antimicrobial effects of rosemary extracts in edible coatings against Pseudomonas spp., lactic acid bacteria, Staphylococcus aureus, Listeria monocytogenes, and Clostridium perfringens, thus ruling this type of extract as a viable solution for incorporation in food coatings (Andrade et al., 2018; Choulitoudi et al., 2017). To the authors' knowledge, there are no previous reports of the effect of rosemary coatings on the microbial quality and safety of cooked ham.

In general, dipped samples followed the same trend of microbial growth as non-treated ("Control") samples. It should be noted that "Control" samples suffered a progressive and significant water loss (superficial dehydration), when compared with dipped samples. As a matter of consequence, dipping the cooked ham in the carrageenan polymer with rosemary extract seems to exert a microbial control effect comparable to that resulting from superficial dehydration.

\subsection{Texture analysis}

Table 1 shows the results obtained for the moisture and texture analysis. They are presented as the mean value of each storage time (ST), regardless of the treatment (T) applied to the ham pieces. The bottom section displays the means and standard deviation of the treatments, regardless of the storage time. This type of statistical procedure intends to show the best treatment ("Control", "Dipping", "Wrapping" or "Both") independently of the storage time (ST), and concomitantly, the best storage time for each can also be visualized, independently of the treatment $(\mathrm{T})$ they endure. Furthermore, the intensity of each factor (ST and T) over each sample can be acknowledged independently. Finally, the interaction of both factors $(\mathrm{ST} \times \mathrm{T}<0.05)$ over the samples is also displayed, which seemed to be the most frequent occurrence. Thus, the standard deviations should not be regarded as a measure of accuracy of the assays, given that they encompass the results of the non-fixed factor (ST and T). Every time a significant interaction was detected (ST $\times \mathrm{T}<0.05$ ), no multiple comparisons could be carried out, meaning that both factors contributed to the observed changes in the samples, thus, the influence of each factor could be evaluated from the estimated marginal means (EMM), present in Figure A1 (supplementary material).

Regarding moisture loss over time, calculated by weighing the samples at the different storage periods, it is clear that there was a loss of water through storage time, although the samples that were subject to the plastic wrap ("Wrapping" and "Both") showed a higher retention of water. The coating solution was not excellent at maintaining the humidity of the ham samples, although it was more satisfactory than 
Table 1

Texture profile and weight variation of the hams subjected to different treatments along the 15-day storage time.

\begin{tabular}{|c|c|c|c|c|c|c|c|c|c|}
\hline & & Weight (g) & Hardness (g) & Adhesiveness (g.sec) & Springiness (\%) & Cohesiveness (\%) & Gumminess & Chewiness & Resilience (\%) \\
\hline \multirow[t]{7}{*}{ Storage time (ST) } & 0 days & $31 \pm 2$ & $2766 \pm 382$ & $-20 \pm 29$ & $2.1 \pm 0.9$ & $0.87 \pm 0.02$ & $3362 \pm 780$ & $7459 \pm 3942$ & $0.49 \pm 0.04$ \\
\hline & 3 days & $28 \pm 3$ & $2890 \pm 856$ & $-8 \pm 5$ & $3 \pm 1$ & $0.86 \pm 0.03$ & $2248 \pm 627$ & $6997 \pm 2459$ & $0.50 \pm 0.38$ \\
\hline & 5 days & $27 \pm 4$ & $3330 \pm 865$ & $-4 \pm 5$ & $3.0 \pm 0.9$ & $0.86 \pm 0.02$ & $2931 \pm 728$ & $8160 \pm 3289$ & $0.50 \pm 0.04$ \\
\hline & 7 days & $26 \pm 5$ & $3573 \pm 726$ & $-8 \pm 13$ & $3.0 \pm 0.9$ & $0.86 \pm 0.01$ & $2850 \pm 869$ & $8935 \pm 4368$ & $0.51 \pm 0.02$ \\
\hline & 10 days & $24 \pm 7$ & $3913 \pm 711$ & $-5 \pm 7$ & $2 \pm 1$ & $0.85 \pm 0.01$ & $3221 \pm 877$ & $6920 \pm 3416$ & $0.49 \pm 0.05$ \\
\hline & 12 days & $24 \pm 7$ & $4380 \pm 709$ & $-5 \pm 7$ & $2 \pm 1$ & $0.84 \pm 0.01$ & $3174 \pm 837$ & $6110 \pm 4144$ & $0.48 \pm 0.04$ \\
\hline & 15 days & $23 \pm 9$ & $4483 \pm 753$ & $-1 \pm 1$ & $3.6 \pm 0.2$ & $0.86 \pm 0.02$ & $3671 \pm 988$ & $7272 \pm 3246$ & $0.49 \pm 0.08$ \\
\hline$p$-value $(\mathrm{n}=42)$ & Tukey's HSD test & $<0.001$ & $<0.001$ & $<0.001$ & $<0.001$ & $<0.001$ & $<0.001$ & $<0.001$ & $<0.001$ \\
\hline$p$-value $(\mathrm{n}=42)$ & Tukey's HSD test & $<0.001$ & $<0.001$ & $<0.001$ & $<0.001$ & $<0.001$ & $<0.001$ & 0.005 & $<0.001$ \\
\hline \multirow[t]{4}{*}{ Treatment (T) } & Control & $21 \pm 5$ & $4352 \pm 1102$ & $-2 \pm 2$ & $2 \pm 1$ & $0.86 \pm 0.02$ & $3459 \pm 926$ & $8365 \pm 3433$ & $0.46 \pm 0.03$ \\
\hline & Dipping & $21 \pm 5$ & $3779 \pm 1298$ & $-3 \pm 3$ & $2 \pm 1$ & $0.85 \pm 0.01$ & $3174 \pm 1026$ & $5408 \pm 2801$ & $0.45 \pm 0.03$ \\
\hline & Wrapping & $31 \pm 2$ & $3187 \pm 584$ & $-9 \pm 12$ & $3 \pm 1$ & $0.87 \pm 0.02$ & $2825 \pm 725$ & $8678 \pm 4009$ & $0.53 \pm 0.02$ \\
\hline & Both & $31 \pm 2$ & $3159 \pm 605$ & $-18 \pm 24$ & $3 \pm 1$ & $0.86 \pm 0.02$ & $2804 \pm 797$ & $7179 \pm 3267$ & $0.54 \pm 0.03$ \\
\hline$p$-value $(\mathrm{n}=24)$ & Tukey's HSD test & $<0.001$ & $<0.001$ & $<0.001$ & $<0.001$ & $<0.001$ & $<0.001$ & $<0.001$ & $<0.001$ \\
\hline $\mathrm{ST} \times \mathrm{T}(\mathrm{n}=168)$ & $p$-value & $<0.001$ & $<0.001$ & $<0.001$ & $<0.001$ & $<0.001$ & $<0.001$ & $<0.001$ & $<0.001$ \\
\hline
\end{tabular}

the "Control" samples, especially between T0 and T7 (Supplementary Material).

Texture analysis consisted of determining various parameters through the TPA test, which consists of a double compression on the foodstuff to mimic the chewing action of the human mouth. The analysed parameters where hardness, adhesiveness, springiness, cohesiveness, chewiness, and resilience. Hardness, defined as the force applied by the molar teeth (Monaco, Cavella, \& Masi, 2008) to compress food showed values between 4483 and $2766 \mathrm{~g}$ of force, with increasing values, over time, revealing that even though there was almost no loss of moisture in the "Wrapping" and "Both" samples, hardness increased over time, probably due to changes carried out by the microbes present on the surface of the hams. Adhesiveness is the ability of food to adhere to the teeth while being chewed (Paula, 2014), and represented as negative values, as its graphical representation is drawn below the " $\mathrm{xx}$ " axis. In the ham samples, the variation occurred between -20 and $-1 \mathrm{~g} \cdot \mathrm{sec}$, thus, inversely to hardness, adhesiveness was reduced over time, with the samples "Wrapping" and "Both" showing higher values, revealing that the moisture content also plays an important role in this textural parameter; even though there is water loss, the adhesiveness was not reduced. This could probably be explained by changes in the nutritional and microbiological composition of the samples, namely an increase in metabolism products of microbes, which have been proven to grow at a higher rate in these samples. Springiness, another important textural parameter which can be defined as the rate at which a deformed food goes back to its undeformed conditions after the removal of the deforming force, so, the rate at which the food goes back to its initial position from the first bite to the second one (Faber, Jaishankar, \& McKinley, 2017). Overall, the ham's springiness was not affected by the different treatments, with very scarce variation between storage time and treatment. Likewise, cohesiveness, related to the springiness of a foodstuff, is defined as the degree to which a food can be deformed before it breaks (Chandra \& Shamasundar, 2015), was also not affected by the storage time or treatment, ranging from 0.85 to $0.87 \%$. Chewiness is the product of gumminess and cohesiveness, being the energy required to masticate the food, and is normally reported for solid foods, while gumminess is used for semi-solid foods, thus not presented in this work (Chandra \& Shamasundar, 2015). Being a product of two other parameters, chewiness has very high variations, and there was no statistically significant change in this parameter during storage time, nor among different treatments. Finally, resilience is the measurement of how a sample recovers from the deformation both in terms of speed and forces, and it seems that for this parameter variation was very low, both among the treatments and storage times (Chandra \& Shamasundar, 2015). Overall, concerning the interaction $(\mathrm{ST} \times \mathrm{T}$ ) of each factor among the different texture parameters and weight, there was a significant interaction ( $\mathrm{p}<0.001$ ) among all, thus, implying that both the storage time and treatment had significant influence on the outcome in terms of texture, not allowing for a marked difference carried out by one individual factor. Still, some conclusions were drawn from the EMM's, and are presented as Figure A1 (supplementary material). For instance, it can be observed on the outputs for hardness, that all samples increased in hardness, with "Control" and "Dipping" showing a higher increase, probably due to a lower moisture content, since these two samples lost a higher amount of moisture over time compared to the "Wrapping" and "Both" samples. This distinction is also visible for the resilience parameter, in which, overtime, the samples enveloped in plastic wrap ("Both" and "Wrapping") showed higher resilience to chewing, while the other two had a lower resilience over time, although the variation is very slight.

\subsection{Colour}

The statistical analysis used for the texture studies was also used to calculate the external colour of the samples, being the analysed parameters $\mathrm{L}^{*}, \mathrm{a}^{*}$ and $\mathrm{b}^{*}$, which are detailed in Table 2. For $\mathrm{L}^{*}$, lightness, a significant interaction was not found $(\mathrm{ST} \times \mathrm{T})$, thus an individual classification for ST and T could be sought. In terms of the factor "Treatment", given that $p$ was higher than 0.05 , no classification could be carried out, while for "Storage Time", the sample with the lowest lightness was the one subject to the dipping treatment, with the "Wrapping" sample being the one with the highest lightness. This implies that there is a reduction in lightness in the samples treated with the natural dipping solution, although this change is a mere 4 points under the "Wrapping" samples, which does not seem very relevant on a scale that ranges from 0 to 100 . In terms of the redness-greenness ( $\left.a^{*}\right)$

Table 2

Color profile $\left(\mathrm{L}^{*}, \mathrm{a}^{*}\right.$, and $\left.\mathrm{b}^{*}\right)$ profile of the hams subjected to different treatments along the 15-day storage time.

\begin{tabular}{lllll}
\hline & & $L^{*}$ & $\mathrm{a}^{*}$ & $\mathrm{~b}^{*}$ \\
\hline Storage time (ST) & 0 days & $64 \pm 3$ & $9 \pm 2$ & $9 \pm 4$ \\
& 3 days & $64 \pm 3$ & $11 \pm 2$ & $9 \pm 2$ \\
& 5 days & $64 \pm 2$ & $10 \pm 2$ & $7 \pm 3$ \\
& 7 days & $64 \pm 3$ & $9 \pm 2$ & $7 \pm 2$ \\
& 10 days & $63 \pm 3$ & $9 \pm 2$ & $10 \pm 4$ \\
& 12 days & $63 \pm 3$ & $10 \pm 1$ & $8 \pm 3$ \\
15 days & $64 \pm 2$ & $10 \pm 2$ & $7 \pm 2$ \\
Treatment (T) & Tukey's HSD test & 0.052 & $<0.001$ & $<0.001$ \\
& Control & $64 \pm 2^{\mathrm{b}}$ & $12 \pm 1$ & $7 \pm 1$ \\
& Dipping & $62 \pm 3^{\mathrm{a}}$ & $9 \pm 1$ & $12 \pm 3$ \\
& Wrapping & $66 \pm 2^{\mathrm{c}}$ & $10 \pm 1$ & $5 \pm 1$ \\
& Both & $64 \pm 3^{\mathrm{b}}$ & $8 \pm 2$ & $8 \pm 2$ \\
-value $(\mathrm{n}=24)$ & Tukey's HSD test & $<0.001$ & $<0.001$ & $<0.001$ \\
ST $\times \mathrm{T}(\mathrm{n}=168)$ & $p$-value & 0.108 & $<0.001$ & $<0.001$ \\
& & & &
\end{tabular}


and yellowness-blueness ( $\mathrm{a}^{*}$ ), there was a significant interaction among the storage time and the treatment undergone by the samples. Thus, only some tendencies where extracted from the estimated marginal means (Figure A1, supplementary material), namely that overall the "Control" sample showed the highest values in redness and maintained them overtime, followed by the "Wrapping" samples. In terms of yellowness-blueness, the sample closer to the yellow section of the colour sphere was the "Dipping" one, while the "Wrapping" had the lowest values, closer to the blueness section. Thus, considering all three-colour axis, although the dipping solution showed darker samples, this reduced the blueness of the samples overtime, placing them closer to the yellow section. Inversely, although the samples treated with plastic "Wrapping" and "Both" showed higher lightness levels, this could partially be explained by the reflective properties of the plastic wrap. Furthermore, "Wrapping" samples were placed closest to the blue section of the colour sphere, which is an undesired colour for meat products, while the wrapped samples placed closer to the redness, which is a desired colour. This maintenance of redness is partially explained by the moisture preservation within the plastic wrap, and although making the ham desirable for its appearance, moisture usually helps increase the microbial load, as verified in section 3.1.

\section{Conclusions}

The aim of this work was to infer the benefits of using bio-based food coatings made from iota-carrageenan as a substitute of plastic wrapping in fresh cooked ham. The microbial load, texture profile and external colour were analysed in specific intervals along a 15-day period. Overall, in terms of microbial load, the samples able to retain moisture over the defined storage time showed the highest count of contaminants, although the sample subjected to dipping in the biobased solution prior to wrapping in plastic had a lower count, proving that the coating had antimicrobial properties. This proves that if the dipping solution fails to be adopted as a standalone solution, it can be successfully used as a co-adjuvant with other preservation methods. Still, for the coliforms, contaminants that pose the highest concerns in terms of food spoilage and safety, the dipping solution had the lowest count, proving its worth in terms of maintaining these contaminants at bay. In all types of microbial load, during the final steps of storage, the dipped samples had the lowest amount, proving that over a long period of time the solution has a higher effect, being this phenomenon mostly observable for APC's, coliforms, and yeasts. Bearing in mind that the samples (two cubic centimetres) are smaller than the full marketed pieces of cooked ham, these results are to be considered as preliminary, but the developed coating solution has proven to be a promising alternative for the food industry, thus, a scale-up of the obtained results will be carried out. Furthermore, 15 days of storage is a very long storage time, that will not be fully used, given European legislation in terms of food preservation. In the first five days of storage, the dipping solution effectively controlled the contaminant growth. The small size of the pieces implied a very low amount of water and consequently a short migration distance from the centre of the pieces to their boundaries. The polymer's low average capacity of avoiding water loss resulted in reduced moisture in the sample, which in turn reduced the activity of the extracts dissolved in it, but also to its film forming ability. This enhanced effect can be observed in Figs. 1-4, in which after the seventh day, the "Wrapping" samples all showed higher growth for all tested microorganisms. Still, considering the inhibition of these microorganisms, rosemary seems to be a very good candidate to be used with ascorbic acid and carrageenan or other biopolymers as a natural sustainable food coating.

In terms of texture analysis, as expected, the higher loss of moisture in the "Control" and "Dipping" samples helped increase their hardness, although the adhesiveness was reduced. Springiness, cohesiveness and resilience did not vary much over time for any of the applied treatment, while there was a high variation for chewiness, although few conclusions could be drawn from this parameter. Lightness was reduced in the samples without plastic wrap over time, although the actual plastic could reflect some of the light. Still, the dipped samples showed a yellow tendency over time, avoiding an increase of blueish colour, which is desirable outcome.

The objectives of developing an alternative solution to plastic wrap were achieved, especially in terms of reducing the microbial load on the surface of the hams. The sustainable and natural coating preserved the samples, ensuring a lower amount of food contaminants, very little colour loss and average changes in texture. The main limitation of the dipping solution is the reduced capacity as a moisture barrier, which can compromise the texture and profile colour. Still, the storage times used in this work go beyond the usual ones in retail and in households, which are around 5-9 days.

\section{Conflicts of interest}

The authors state no conflict of interest.

\section{Acknowledgments}

The authors are grateful to the Foundation for Science and Technology (FCT, Portugal and FEDER, under Program PT2020 for financial support to CIMO (UID/AGR/00690/2013); to project POCI-010145-FEDER-006984 - Associate Laboratory LSRE-LCM funded by FEDER through COMPETE2020-POCI and by FCT; M. Carocho (SFRH/ BPD/114650/2016) and S.A. Heleno (SFRH/BPD/101413/2014) postdoctoral grants and L. Barros' contract. This work is funded by the European Structural and Investment Funds (FEEI) through the Regional Operational Program North 2020, within the scope of Project NORTE01-0145-FEDER-023289: DeCodE. The authors thank "SONAE MC" for the donation of the ham piece that were used in this work.

\section{Appendix A. Supplementary data}

Supplementary data to this article can be found online at https:// doi.org/10.1016/j.lwt.2019.01.031.

\section{References}

Absalan, F., Saremy, S., Mansouri, E., Moghadam, M. T., Moghadam, A. R. E., \& Ghanavati, R. (2017). Effects of mono-(2-ethylhexyl) phthalate and di-(2-ethylhexyl) phthalate administrations on oocyte meiotic maturation, apoptosis and gene quantification in mouse model. Cell Journal, 18, 503-513.

Aloui, H., \& Khwaldia, K. (2016). Natural antimicrobial edible coatings for microbial safety and food quality enhancement. Comprehensive Reviews in Food Science and Food Safety, 15, 1080-1103.

Andrade, M. A., Ribeiro-Santos, R., Bonito, M. C. C., Saraiva, M., \& Sanches-Silva, A. (2018). Characterization of rosemary and thyme extracts for incorporation into a whey protein based film. LWT-Food Science and Technology, 92, 497-508.

Brostow, W., Lu, X., \& Osmanson, A. T. (2018). Nontoxic bio-plasticizers for PVC as replacement for conventional toxic plasticizers. Polymer Testing, 69, 63-70.

Carocho, M., \& Ferreira, I. C. F. R. (2013). A review on antioxidants, prooxidants and related controversy: Natural and synthetic compounds, screening and analysis methodologies and future perspectives. Food and Chemical Toxicology, 51, 15-25.

Chandra, M. V., \& Shamasundar, B. A. (2015). Texture profile analysis and functional properties of gelatin from the skin of three species of fresh water fish. International Journal of Food Properties, 18, 572-584.

Chong, J. X., Lai, S., \& Yang, H. (2015). Chitosan combined with calcium chloride impacts fresh-cut honeydew melon by stabilising nanostructures of sodium-carbonate-soluble pectin. Food Control, 53, 195-205.

Choulitoudi, E., Ganiari, S., Tsironi, T., Ntzimani, A., Tsimogiannis, D., Toukis, P., et al. (2017). Edible coating enriched with rosemary extracts to enhance oxidative and microbial stability of smoked eel fillets. Food Packaging and Shelf Life, 12, 107-113.

Dehghani, S., Hosseini, S. V., \& Regenstein, J. M. (2018). Edible films and coatings in seafood preservation: A review. Food Chemistry, 240, 505-513.

EFSA (2008). Use of rosemary extracts as a food additive. The EFSA Journal, 721, 1-29.

Faber, T. J., Jaishankar, A., \& McKinley, G. H. (2017). Describing the firmness, springiness and rubberiness of food gels using fractional calculus. Part I: Theoretical framework. Food Hydrocolloids, 62, 311-324.

Ganiari, S., Choulitoudi, E., \& Oreopoulou, V. (2017). Edible and active films and coatings as carriers of natural antioxidants for lipid food. Trends in Food Science \& Technology, 68, 70-82. 
Gonçalves, G. A., Corrêa, R. C. G., Barros, L., Dias, M. I., Calhelha, R. C., Correa, V. G., et al. (2019). Effects of in vitro gastrointestinal digestion and colonic fermentation on a rosemary (Rosmarinus officinalis L.) extract rich in rosmarinic acid. Food Chemistry, 271, 393-400.

ISO (1998). ISO 15214:1998- Microbiology of food and animal feeding stuffs - Horizontal method for the enumeration of mesophilic lactic acid bacteria - Colony-count technique at 30 degrees C. International Organization for Standardization.

ISO (1999). ISO 6888-1:1999- Microbiology of food and animal feeding stuffs - Horizontal method for the enumeration of coagulase-positive staphylococci (Staphylococcus aureus and other species) - Part 1: Technique using Baird-Parker agar medium. International Organization for Standardization.

ISO (2003). ISO 15213 Microbiology of food and animal feeding stuffs - Horizontal method for the enumeration of sulfite- reducing bacteria growing under anaerobic conditions.

ISO (2006). ISO 4832 Microbiology of food and animal feeding stuffs - Horizontal method for the enumeration of coliforms - Colony-count technique.

ISO (2008). ISO 21527-1/2:2008 - microbiology of food and animal feeding stuffs horizontal method for the enumeration of yeasts and moulds. In ISO copyright Office (Ed.). International standardization organization(1st ed.). (Switzerland).

ISO (2013). ISO 4833-1 Microbiology of the food chain - horizontal method for the enumeration of microorganisms-Part 1: Colony count at 30 degrees $C$ by the pour plate technique.

Jiang, Z., Neetoo, H., Chen, \& Haiqiang (2011). Efficacy of freezing, frozen storage and edible antimicrobial coatings used in combination for control of Listeria monocytogenes on roasted Turkey stored at chiller temperatures. Food Microbiology, 28 1394-1401.

Kharchoufi, S., Parafiti, L., Licciardello, F., Muratore, G., Hamdi, M., Cirvilleri, et al. (2018). Edible coatings incorporating pomegranate peel extract and biocontrol yeast to reduce Penicillium digitatum postharvest decay of oranges. Food Microbiology, 74, 107-112.

Lopez, D. S., Advani, S., Tsilidis, K. K., Wang, R., Baillargeon, J., Dobs, A., et al. (2017). Association of urinary phthalate metabolites with erectile dysfunction in racial and ethnic groups in the national health and nutrition examination survey 2001-2004. American Journal of Men's Health, 11, 576-584.

Monaco, R. D., Cavella, S., \& Masi, P. (2008). Predicting the sensory cohesiveness, hardness and springiness of solid foods from instrumental measurements. Journal of Texture Studies, 39, 129-149.

Muncke, J., Backhaus, T., Geueke, B., Maffini, M. V., Martin, O. V., Meyers, J. P., et al (2017). Scientific challenges in the risk assessment of food contact materials. Environmental Health Perspectives095001 1-9.
Nerin, C., Canellas, E., \& Vera, P. (2018). Plasticizer migration into foods. Reference Models in Food Science. https://doi.org/10.1016/B978-0-08-100596-5.21392-9 Elsevier.

Paula, A. M. (2014). Texture profile and correlation between sensory and instrumental analyses on extruded snacks. Journal of Food Engineering, 121, 9-14.

Pereira, J. O., Soares, J., Monteiro, M. J. P., Gomes, A., \& Pintado, M. (2018). Impact of whey protein coating incorporated with Bifidobacterium and Lactobacillus on sliced ham properties. Meat Science, 139, 125-133.

Portillo, H. A., Howlander, M. S., Campbell, Y. L., French, T., Kim, T., Goddard, J., et al. (2018). Incorporating fermented by-products of Lactobacillus diolivorans in food grade coatings designed for inhibition of Tyrophagus putrescentiae on dry-cured hams. Journal of Stored Products Research, 77, 77-83.

Ribeiro, A., Caleja, C., Barros, L., Santos-Buelga, C., Barreiro, M. F., \& Ferreira, I. C. F. R. (2016). Rosemary extracts in functional foods: Extraction, chemical characterization and incorporation of free and microencapsulated forms in cottage cheese. Food \& Function, 7, 2185-2196.

Rocha, B. A., Asimakopoulos, A. G.,, Jr., F.B, \& Kannan, K. (2017). Urinary concentrations of 25 phthalate metabolites in Brazilian children and their association with oxidative DNA damage. The Science of the Total Environment, 586, 152-162.

Ruiz-Navajas, Y., Viuda-Martos, M., Barber, X., Sendra, E., Perez-Alvarez, J. A., \& Fernández-López, J. (2015). Effect of chitosan edible films added with Thymus moroderi and Thymus piperella essential oil on shelf-life of cooked cured ham. Journal of Food Science \& Technology, 52, 6493-6501.

Stojanoska, M. M., Milosevic, N., Milic, N., \& Abenavoli, L. (2017). The influence of phthalates and bisphenol A on the obesity development and glucose metabolism disorders. Endocrine, 55, 666-681.

Tavassoli-Kafrani, E., Shekarchizadeh, H., \& Masoudpour-Behabadi, M. (2016). Development of edible films and coatings from alginates and carrageenans. Carbohydrate Polymers, 137, 360-374.

Toni, L. D., Tisato, F., Seraglia, R., Roverso, M., Gandin, V., Marzano, C., et al. (2017). Phthalates and heavy metals as endocrine disruptors in food: A study on pre-packed coffee products. Toxicology Reports, 4, 234-239.

Viuda-Martos, M., Ruiz-Navajas, Y., Fernández-López, J., \& Péres-Álvarez, J. A. (2010). Effect of added citrus fibre and spice essential oils on quality characteristic and shelflife of mortadella. Meat Science, 568-576.

Zuccarello, P., Conti, G. O., Cavallaro, F., Copat, C., Cristaldi, C., Fiore, M., et al. (2018) Implication of dietary phthalates in breast cancer. A systematic review. Food and Chemical Toxicology, 118, 667-674. 